\title{
Living Well with Kidney Disease by Patient and Care-Partner Empowerment: Kidney Health for Everyone Everywhere
}

\author{
Kamyar Kalantar-Zadeh ${ }^{\mathrm{a}}$ Philip Kam-Tao Li ${ }^{\mathrm{b}}$ Ekamol Tantisattamo ${ }^{\mathrm{c}}$ Latha Kumaraswami $^{\mathrm{d}}$ \\ Vassilios Liakopoulos ${ }^{\text {e }}$ Siu-Fai Lui ${ }^{f}$ Ifeoma Ulasi ${ }^{9}$ Sharon Andreoli ${ }^{\text {h }}$ Alessandro Balducci ${ }^{i}$ \\ Sophie Dupuis ${ }^{j}$ Tess Harris ${ }^{k}$ Anne Hradskyj ${ }^{j}$ Richard Knight ${ }^{l}$ Sajay Kumard Maggie Ng ${ }^{\mathrm{m}}$ \\ Alice Poidevin ${ }^{j}$ Gamal Saadi $^{\mathrm{n}}$ Allison Tong ${ }^{\circ}$ for the World Kidney Day Steering Committee \\ ${ }^{a}$ The International Federation of Kidney Foundation - World Kidney Alliance (IFKF-WKA), Division of Nephrology \\ and Hypertension and Kidney Transplantation, University of California Irvine, Orange, CA, USA; ${ }^{b}$ Department of \\ Medicine and Therapeutics, Carol and Richard Yu PD Research Centre, Prince of Wales Hospital, Chinese University \\ of Hong Kong, Hong Kong, China; ' Division of Nephrology, Hypertension and Kidney Transplantation, Department \\ of Medicine, University of California Irvine School of Medicine, Orange, CA, USA; ${ }^{\mathrm{d} T a n k e r ~ F o u n d a t i o n, ~ C h e n n a i, ~}$

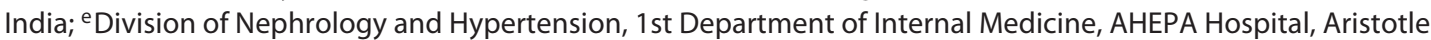 \\ University of Thessaloniki, Thessaloniki, Greece; ${ }^{f}$ Hong Kong Kidney Foundation and the International Federation \\ of Kidney Foundations - World Kidney Alliance, The Jockey Club School of Public Health and Primary Care, The \\ Chinese University of Hong Kong, Hong Kong, China; ${ }^{9}$ Renal Unit, Department of Medicine, College of Medicine,

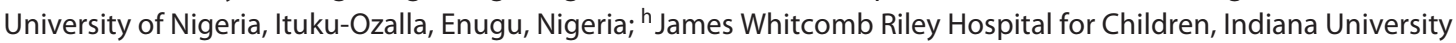 \\ School of Medicine, Indianapolis, IN, USA; 'Italian Kidney Foundation, Rome, Italy; 'World Kidney Day Office,

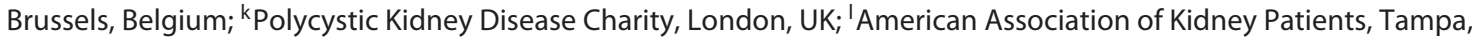 \\ FL, USA; ${ }^{m}$ Hong Kong Kidney Foundation, Hong Kong, China; ${ }^{n}$ Nephrology Unit, Department of Internal Medicine, \\ Faculty of Medicine, Cairo University, Giza, Egypt; ${ }^{\circ}$ Sydney School of Public Health, The University of Sydney, \\ Sydney, NSW, Australia
}

\section{Keywords}

Patient empowerment · Care-partner · Low-middle-income countries $\cdot$ Health policy

This article is being published in Kidney International and reprinted concurrently in several journals. The articles cover identical concepts and wording, but vary in minor stylistic and spelling changes, detail, and length of manuscript in keeping with each journal's style. Any of these versions may be used in citing this article. Note that all authors contributed equally to the conception, preparation, and editing of the manuscript.

Members of the World Kidney Day Steering Committee are listed in the Appendix.

karger@karger.com www.karger.com/kdd

Karger $\stackrel{\text { ' }}{5}$

GOPEN ACCESS (c) 2021 World Kidney Day 2021 Steering Committee

This is an Open Access article licensed under the Creative Commons Attribution-NonCommercial-4.0 International License (CC BY-NC) (http://www.karger.com/Services/OpenAccessLicense), applicable to the online version of the article only. Usage and distribution for commercial purposes requires written permission.

\section{Abstract}

Living with chronic kidney disease (CKD) is associated with hardships for patients and their care-partners. Empowering patients and their care-partners, including family members or friends involved in their care, may help minimize the burden and consequences of CKD-related symptoms to enable life participation. There is a need to broaden the focus on living well with kidney disease and re-engagement in life, including an emphasis on patients being in control. The World Kidney Day (WKD) Joint Steering Committee has declared 2021 the year of "Living Well with Kidney Disease" in an effort to increase education and awareness on the impor-
Correspondence to:

Kamyar Kalantar-Zadeh, kkz@uci.edu Philip Kam-Tao Li, philipli@ cuhk.edu.hk 
tant goal of patient empowerment and life participation. This calls for the development and implementation of validated patient-reported outcome measures to assess and address areas of life participation in routine care. It could be supported by regulatory agencies as a metric for quality care or to support labelling claims for medicines and devices. Funding agencies could establish targeted calls for research that address the priorities of patients. Patients with kidney disease and their care-partners should feel supported to live well through concerted efforts by kidney care communities, including during pandemics. In the overall wellness program for kidney disease patients, the need for prevention should be reiterated. Early detection with a prolonged course of wellness despite kidney disease, after effective secondary and tertiary prevention programs, should be promoted. WKD 2021 continues to call for increased awareness of the importance of preventive measures throughout populations, professionals, and policy makers, applicable to both developed and developing countries.

๑) 2021 World Kidney Day 2021 Steering Committee

\section{Patient Priorities for Living Well: A Focus on Life Participation}

Chronic kidney disease (CKD), its associated symptoms, and its treatment, including medications, dietary and fluid restrictions, and kidney replacement therapy can disrupt and constrain daily living, and impair the overall quality of life of patients and their family members. Consequently, this can also impact treatment satisfaction and clinical outcomes [1]. Despite this, the past several decades have seen limited improvement in the quality of life of people with CKD [1]. To advance research, practice, and policy, there is increasing recognition of the need to identify and address patient priorities, values, and goals [1].

Several regional and global kidney health projects have addressed these important questions including the Standardised Outcomes in Nephrology (SONG) with more than 9,000 patients, family members, and health professionals from over 70 countries $[2,3]$. Across all treatment stages, including $\mathrm{CKD}$, dialysis and transplantation, SONG-participating children and adults with CKD consistently gave higher priority to symptoms and life impacts than health professionals $[2,3]$. In comparison, health professionals gave higher priority to mortality and hospitalization than patients and family members. The patient-prioritized outcomes are shown in Figure 1. Irrespective of the type of kidney disease or treatment stage, patients wanted to be able to live well, maintain their role and social functioning, protect some semblance of normality, and have a sense of control over their health and wellbeing.

Life participation, defined as the ability to do meaningful activities of life including, but not limited to, work, study, family responsibilities, travel, sport, social and recreational activities, was established a critically important outcome across all treatment stages of CKD $[1,2]$. The quotations from patients with kidney disease provided in Box 1 demonstrate how life participation reflects the ability to live well with CKD [4]. According to the World Health Organization (WHO), participation refers to "involvement in a life situation" [5]. This concept is more specific than the broader construct of quality of life. Life participation places the life priorities and values of those affected by CKD and their family at the center of decision making. The World Kidney Day (WKD) Steering Committee calls for the inclusion of life participation, a key focus in the care of patients with $\mathrm{CKD}$, to achieve the ultimate goal of living well with kidney disease. This calls for the development and implementation of validated patient-reported outcome measures that could be used to assess and address areas of life participation in routine care. Monitoring of life participation could be supported by regulatory agencies as a metric for quality care or to support labelling claims for medicines and devices. Funding agencies could establish targeted calls for research that address the priorities of patients, including life participation.

\section{Patient Empowerment, Partnership, and a Paradigm Shift towards a Strengths-Based Approach to Care}

Patients with CKD and their family members including care-partners should be empowered to achieve the health outcomes and life goals that are meaningful and important to them. The WHO defines patient empowerment as "a process through which people gain greater control over decisions or actions affecting their health" [6], which requires patients to understand their role, to have knowledge to be able to engage with clinicians in shared decision-making, skills, and support for self-management. For patients receiving dialysis, understanding the rationale for a lifestyle change, having access to practical assistance and family support promoted patient empowerment, while feeling limited in life participation undermined their sense of empowerment [7].

The WKD Steering Committee advocates for strengthened partnership with patients in the development, im- 


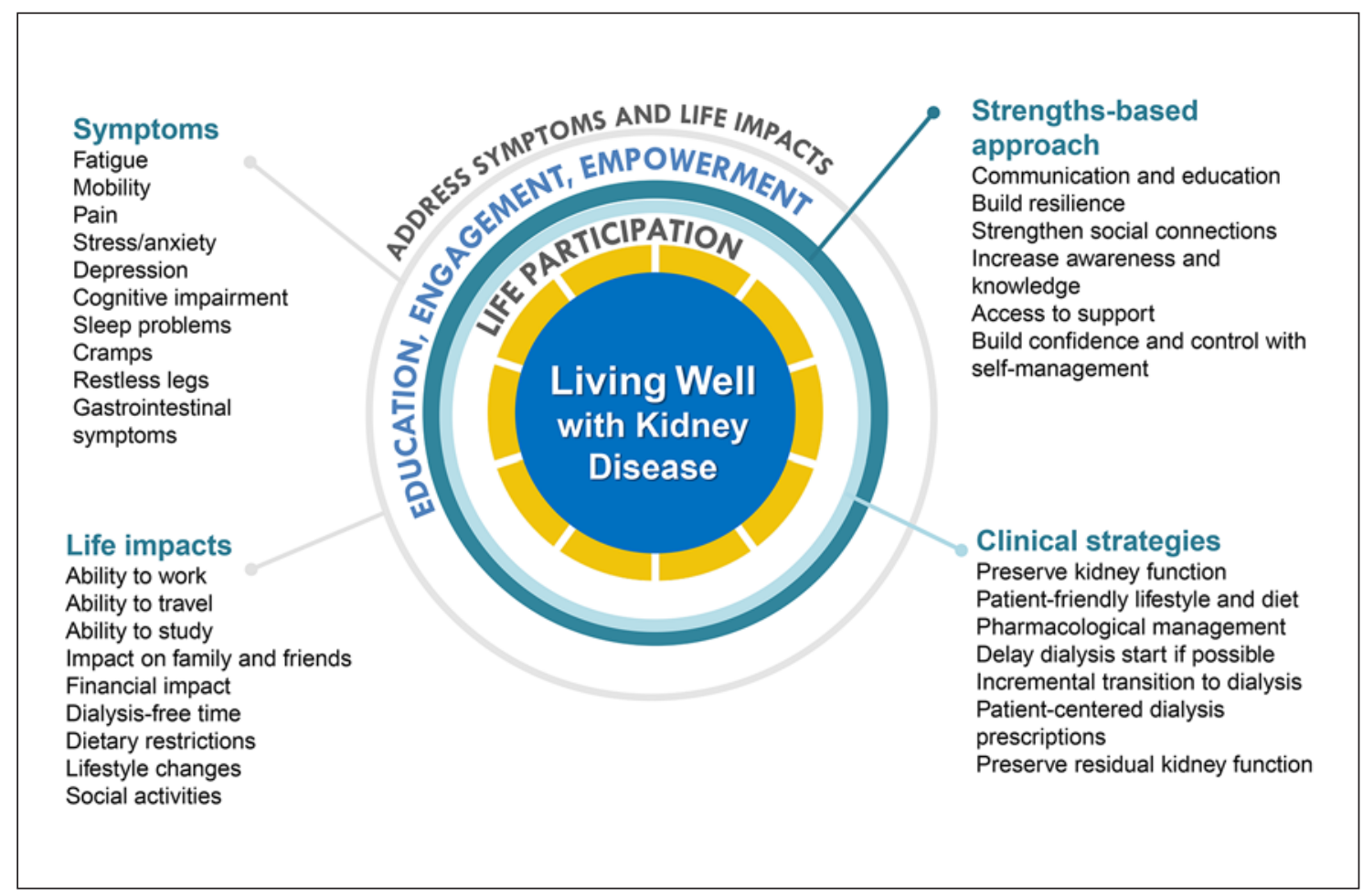

Fig. 1. Conceptual framework of "Living Well with Kidney Disease" based on patient centeredness and empowering patients with focus on effective symptom management and life participation.

plementation, and evaluation of interventions for practice and policy settings that enable patients to live well with kidney diseases. This needs to be supported by consistent, accessible, and meaningful communication. Meaningful involvement of patients and family members across the entire research process, from priority setting and planning the study through to dissemination and implementation, is now widely advocated [8]. There have also been efforts, such as the Kidney Health Initiative, to involve patients in the development of drugs and devices to foster innovation [9].

We urge for greater emphasis on a strengths-based approach, as outlined in Table 1, which encompasses strategies to support patient resilience, harness social connections, build patient awareness and knowledge, facilitate access to support, and establish confidence and control in self-management. The strengths-based approach is in contrast to the medical model where chronic disease is traditionally focused on pathology, problems, and failures [10]. Instead, the strengths-based approach acknowledges that each individual has strengths and abilities to overcome the problems and challenges faced, and re- quires collaboration and cultivation of the patient's hopes, aspirations, interests, and values. Efforts are needed to ensure that structural biases, discrimination, and disparities in the health care system also need to be identified, so all patients are given the opportunity to have a voice.

\section{The Role of Care-Partner}

A care-partner is often an informal caregiver who is also a family member of the patient with CKD [11]. They may take on a wide range of responsibilities including coordinating care (including transportation to appointments), administration of treatment including medications, home dialysis assistance, and supporting dietary management. Caregivers of patients with CKD have reported depression, fatigue, isolation, and also burn out. The role of the care-partner has increasingly become more important in CKD care given the heightened complexity in communicative and therapeutic options including the expansion of telemedicine under the COVID-19 pandemic and given the goal to achieve higher life expectancy with 
Box 1. Quotations from patients with CKD related to priorities for living well

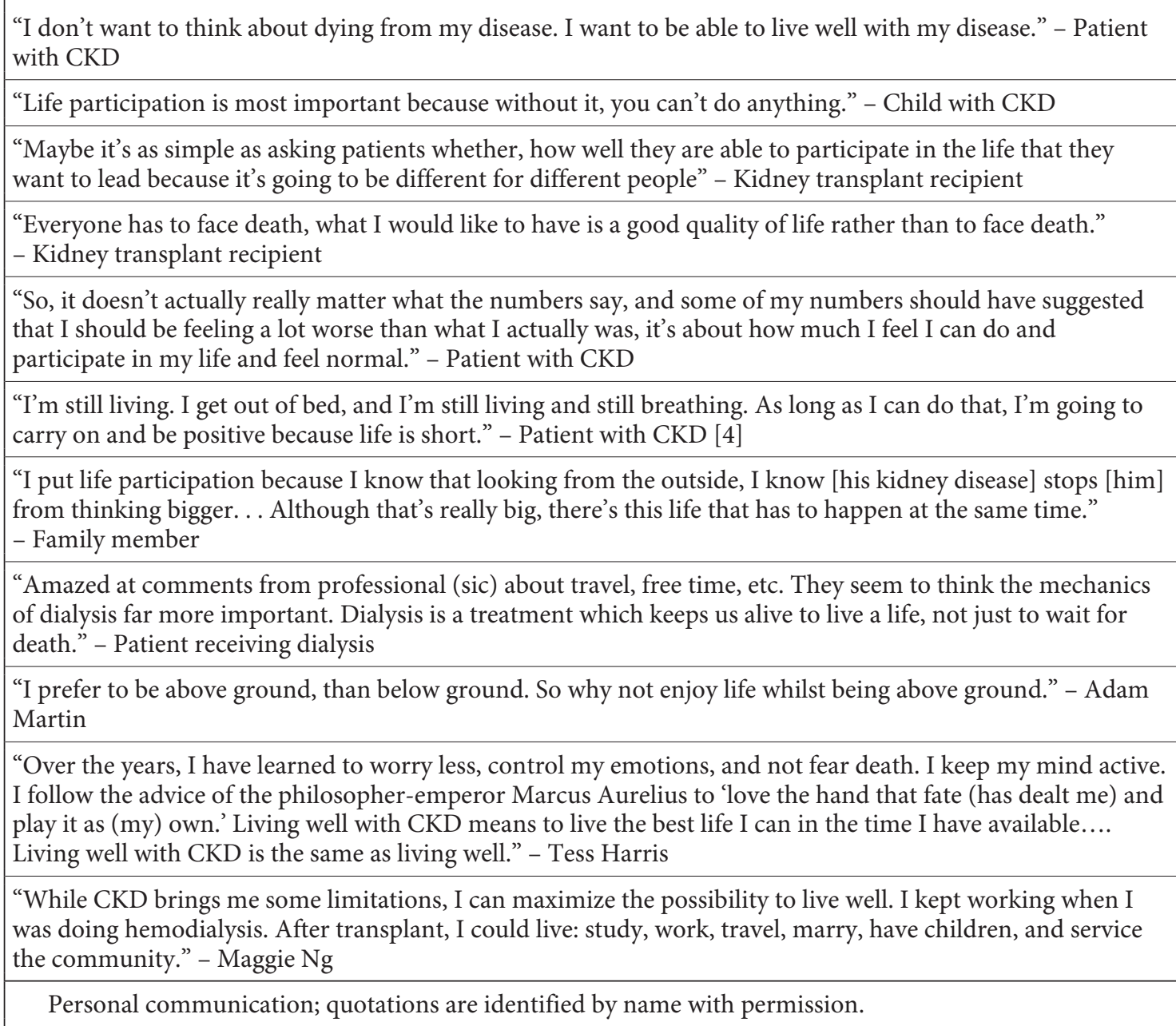

CKD [12]. The experience of caring for a partially incapacitated family member with progressive CKD can represent a substantial burden on the care-partner and may impact family dynamics. Not infrequently, the career goals and other occupational and leisure aspects of the life of the care-partner are affected because of CKD care partnership, leading to care-partner overload and burnout. Hence, the above-mentioned principles of life participation need to equally apply to care-partners as well as all family members and friends involved in CKD care.

\section{Living with Kidney Disease in Low-Income Regions}

In low and low-middle-income countries (LICs and LMICs) including in sub-Saharan Africa, South East Asia, and Latin America, patient's ability to self-manage or cope with the chronic disease vary but may often be influenced by internal factors including spirituality, belief system, and religiosity, and external factors including appropriate knowledge of the disease, poverty, family support system, and one's grit and social relations network. The support system comprising health care providers and caregivers plays a crucial role as most patients rely on them in making decisions, and for the necessary adjustments in their health behavior [13]. In LIC regions, where there are often a relatively low number of physicians and even lower number of kidney care providers per population especially in rural areas, a stepwise approach can involve local and national stakeholders including both nongovernmental organizations and government agencies by: (1) extending kidney patient education in rural areas, (2) adapting telehealth technologies if feasible to educate patients and train local community kidney care provid- 
Table 1. Suggested strategies for "living well with CKD" using a strengths-based approach

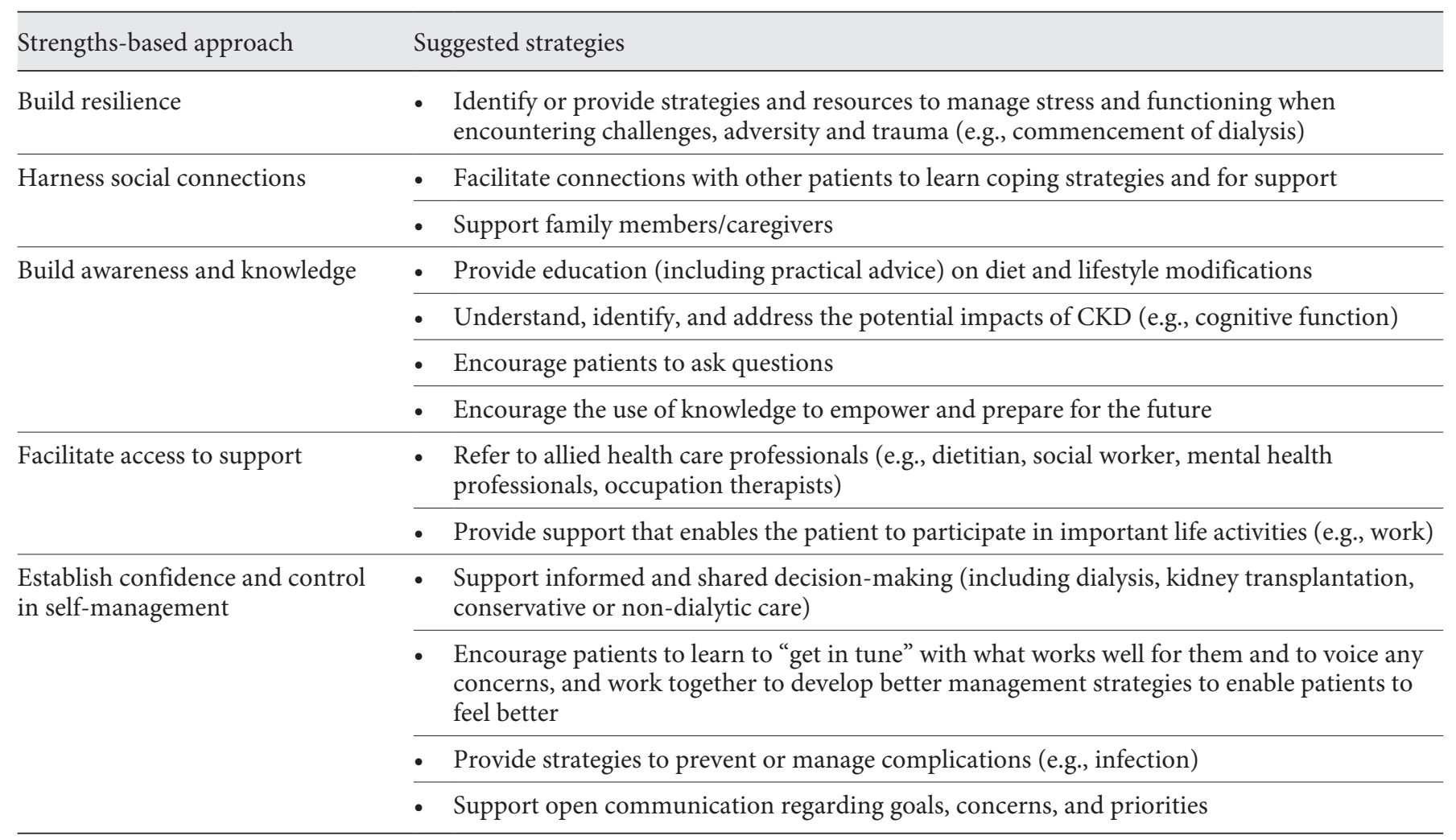

CKD, chronic kidney disease (not receiving kidney replacement therapy).

ers, and (3) implementing effective retention strategies for rural kidney health providers including adapting career plans and competitive incentives.

Many patients in low resource settings present in very late stage needing to commence emergency dialysis [14]. The very few fortunate ones to receive kidney transplantation may acquire an indescribable chance to normal life again, notwithstanding the high costs of immunosuppressive medications in some countries. For some patients and care-partners in low-income regions, spirituality and religiosity may engender hope, when ill they are energized by the anticipation of restored health and spiritual wellbeing. For many patients, informing them of a diagnosis of kidney disease is a harrowing experience both for the patient (and caregivers) and the health care professional. Most patients present to kidney physicians (usually known as "renal physicians" in many of these countries) with trepidations and apprehension. It is rewarding therefore to see the patient's anxiety dissipate after reassuring him or her of a diagnosis of simple kidney cysts, urinary tract infection, simple kidney stones, solitary kidneys, etc., that would not require extreme measures like kidney replacement therapy. Patients diagnosed with glomerulonephritis who have an appropriate characterization of their disease from kidney biopsies and histology, who receive appropriate therapies and achieve remission are relieved and are very grateful. Patients are glad to discontinue dialysis following resolution of AKI or acute on CKD.

Many CKD patients who have residual kidney function appreciate being maintained in a relatively healthy state with conservative measures, without dialysis. They experience renewed energy when their anemia is promptly corrected using erythropoiesis-stimulating agents. They are happy when their peripheral edema resolves with treatment. For those on maintenance hemodialysis who had woeful stories from emergency femoral cannulations, they appreciate the construction of good temporary or permanent vascular accesses. Many patients in lowresource settings present in very late stage needing to commence emergency dialysis. Patients remain grateful for waking from a uremic coma or recovering from recurrent seizures when they commence dialysis. 


\section{World Kidney Day 2021 Advocacy}

The WKD 2021 theme on "Living Well with Kidney Disease" is deliberately chosen to have the goals to redirect more focus on plans and actions towards achieving patient-centered wellness. "Kidney Health for Everyone, Everywhere" with emphasis on patient-centered wellness should be a policy imperative that can be successfully achieved if policy makers, nephrologists, health care professionals, patients, and care partners place this within the context of comprehensive care. The requirement of patient engagement is needed. The WHO in 2016 put out an important document on patient empowerment: "Patient engagement is increasingly recognized as an integral part of health care and a critical component of safe peoplecentered services. Engaged patients are better able to make informed decisions about their care options. In addition, resources may be better used if they are aligned with patients' priorities and this is critical for the sustainability of health systems worldwide. Patient engagement may also promote mutual accountability and understanding between patients and health care providers. Informed patients are more likely to feel confident to report both positive and negative experiences and have increased concordance with mutually agreed care management plans. This not only improves health outcomes but also advances learning and improvement while reducing adverse events." In the ISN Community Film Event at World Congress of Nephrology (WCN) 2020, it is good to see a quote in the film from patients: "Tell me. I will forget; Show me. I will remember; Involve me. I will understand." ISN Global Kidney Policy Forum 2019 included a patient speaker Nicki Scholes-Robertson from New Zealand: "Culturally appropriate and sensitive patient information and care are being undertaken in New Zealand to fight inequities in kidney health, especially in Maori and other disadvantaged communities."

WKD 2021 would like to promote to the policy makers on increasing focus and resources on both drug and nondrug programs in improving patient wellness. Examples include funding for erythropoiesis-stimulating agents and anti-pruritic agents for managing anemia and itchiness, respectively, just to name but a few $[15,16]$. Home dialysis therapies have been consistently found to improve patient autonomy and flexibility, quality of life in a cost-effective manner, enhancing life participation. Promoting home dialysis therapies should tie in with appropriate "assisted dialysis" programs to reduce patient and care partner fatigue and burnout. Also, examples like selfmanagement programs, cognitive behavioral therapy, and group therapies for managing depression, anxiety, and insomnia should be promoted before resorting to medications [17]. The principle of equity recognizes that different people with different levels of disadvantage require different approaches and resources to achieve equitable health outcomes. The kidney community should push for adapted care guidelines for vulnerable and disadvantaged populations. The involvement of primary care and general physicians especially in LICs and LMICs would be useful in improving the affordability and access to services through the public sector in helping the symptom management of CKD patients and improve their wellness. In the overall wellness program for kidney disease patients, the need for prevention should be reiterated. Early detection with a prolonged course of wellness despite kidney disease, after an effective secondary prevention program, should be promoted [18]. Prevention of CKD progression can be attempted by lifestyle and diet modifications such as a plant-dominant low protein diet and by means of effective pharmacotherapy including administration of sodium-glucose transport protein 2 (SGLT2) inhibitors [19]. WKD 2021 continues to call for increased awareness of the importance of preventive measures throughout populations, professionals, and policy makers, applicable to both developed and developing countries [18].

\section{Conclusions}

Effective strategies to empower patients and their carepartners strive to pursue the overarching goal of minimizing the burden of CKD-related symptoms in order to enhance patient satisfaction, health-related quality of life, and life participation. The WKD 2021 theme on "Living Well with Kidney Disease" is deliberately chosen to have the goals to redirect more focus on plans and actions towards achieving patient-centered wellness. Notwithstanding the COVID-19 pandemic that had overshadowed many activities in 2020 and beyond, the WKD Steering Committee has declared 2021 the year of "Living well with Kidney Disease" in an effort to increase education and awareness on the important goal of effective symptom management and patient empowerment. Whereas the WKD continues to emphasize the importance of effective measures to prevent kidney disease and its progression [18], patients with preexisting kidney disease and their care-partners should feel supported to live well through concerted efforts by kidney care communities and other stakeholders throughout the world even 
during a world-shattering pandemic as COVID-19 that may drain many resources [20]. Living well with kidney disease is an uncompromisable goal of all kidney foundations, patient groups, and professional societies alike, to which the International Society of Nephrology and the International Federation of Kidney Foundation World Kidney Alliance are committed at all times.

\section{Conflict of Interest Statement}

K.K.-Z. reports honoraria from Abbott, Abbvie, ACI Clinical, Akebia, Alexion, Amgen, Ardelyx, Astra-Zeneca, Aveo, BBraun, Cara Therapeutics, Chugai, Cytokinetics, Daiichi, DaVita, Frese- nius, Genentech, Haymarket Media, Hospira, Kabi, Keryx, Kissei, Novartis, Pfizer, Regulus, Relypsa, Resverlogix, Dr Schaer, Sandoz, Sanofi, Shire, Vifor, UpToDate, and ZS-Pharma. P.K.T.L. is Editorial Board Member of Kidney Diseases and reports personal fees from Fibrogen and Astra-Zeneca. G.S. reports personal fees from Multicare, Novartis, Sandoz, and Astra-Zeneca. V.L. reports nonfinancial support from Genesis Pharma.

\section{Appendix}

Members of the World Kidney Day Steering Committee are: Philip Kam Tao Li, Kamyar Kalantar-Zadeh, Sharon Andreoli, Alessandro Balducci, Sophie Dupuis, Latha Kumaraswami, Vassilios Liakopoulos, Siu-Fai Lui, Gamal Saadi, and Ifeoma Ulasi.

\section{References}

1 Tong A, Manns B, Wang AY, Hemmelgarn B, Wheeler DC, Gill J, et al.; SONG Implementation Workshop Investigators. Implementing core outcomes in kidney disease: report of the Standardized Outcomes in Nephrology (SONG) implementation workshop. Kidney Int. 2018 Dec;94(6):1053-68. Available from: https://www.ncbi.nlm.nih.gov/pubmed/ 30360959.

2 Carter SA, Gutman T, Logeman C, Cattran D, Lightstone L, Bagga A, et al.; SONG-GD Investigators. Identifying Outcomes Important to Patients with Glomerular Disease and Their Caregivers. Clin J Am Soc Nephrol. 2020 May; 15(5):673-84. Available from: https://www.ncbi.nlm.nih.gov/pubmed/ 32354728.

3 Hanson CS, Craig JC, Logeman C, Sinha A, Dart A, Eddy AA, et al. Establishing core outcome domains in pediatric kidney disease: report of the Standardized Outcomes in $\mathrm{Ne}$ phrology-Children and Adolescents (SONGKIDS) consensus workshops. Kidney Int. 2020; 98(3):553-65. doi: https://doi.org/ 10.1016/j.kint.2020.05.054.

4 Gonzalez AM, Gutman T, Lopez-Vargas P, Anumudu S, Arce CM, Craig JC, Dunn L, Eckardt KU, Harris T, Levey AS, Lightstone L, Scholes-Robertson N, Shen JI, Teixeira-Pinto A, Wheeler DC, White D, Wilkie M, Jadoul M, Winkelmayer WC, Tong A. Patient and caregiver priorities for outcomes in CKD: a multinational nominal group technique study. Am J Kidney Dis. 2020;76(5):679-89. doi: https:// doi.org/10.1053/j.ajkd.2020.03.022.

5 WHO. Towards a common language for functioning, disability and health. EIP/GPE/ CAS/01.3. WHO; 2002

6 WHO. Health promotion glossary. HPR/ HEP/98.1. WHO; 1998.

7 Baumgart A, Manera KE, Johnson DW, Craig JC, Shen JI, Ruiz L, et al. Meaning of empowerment in peritoneal dialysis: focus groups with patients and caregivers. Nephrol Dial Transplant. 2020;35(11):1949-58.
8 PCORI. The Value of Engagement (Available at https://www.pcori.org/about-us/our-programs/engagement/public-and-patient-engagement/value-engagement, accessed 1 st September 2020). 2018.

9 Bonventre JV, Hurst FP, West M, Wu I, RoyChaudhury P, Sheldon M. A Technology Roadmap for Innovative Approaches to Kidney Replacement Therapies: A Catalyst for Change. Clin J Am Soc Nephrol. 2019 Oct; 14(10):1539-47. Available from: https:// www.ncbi.nlm.nih.gov/pubmed/31562182.

10 Ibrahim N, Michail M, Callaghan P. The strengths based approach as a service delivery model for severe mental illness: a meta-analysis of clinical trials. BMC Psychiatry. 2014 Aug; 14(1):243. Available from: https://www. ncbi.nlm.nih.gov/pubmed/25189400.

11 Parham R, Jacyna N, Hothi D, Marks SD, Holttum S, Camic P. Development of a measure of caregiver burden in paediatric chronic kidney disease: The Paediatric Renal Caregiver Burden Scale. J Health Psychol. 2016; 21(2):193-205. doi: https://doi.org/10.1177/ 1359105314524971.

12 Subramanian L, Kirk R, Cuttitta T, Bryant N, Fox K, McCall M, et al. Remote Management for Peritoneal Dialysis: A Qualitative Study of Patient, Care Partner, and Clinician Perceptions and Priorities in the United States and the United Kingdom. Kidney Med. 2019 Oct; 1(6):354-65. Available from: https://www. ncbi.nlm.nih.gov/pubmed/32734216.

13 Angwenyi V, Aantjes C, Kajumi M, De Man J, Criel B, Bunders-Aelen J. Patients experiences of self-management and strategies for dealing with chronic conditions in rural Malawi. PLoS One. 2018 Jul;13(7):e0199977. Available from: https://www.ncbi.nlm.nih. gov/pubmed/29965990.
14 Ulasi II, Ijoma CK. The enormity of chronic kidney disease in Nigeria: the situation in a teaching hospital in South-East Nigeria. J Trop Med. 2010;2010:501957. Available from: https://www.ncbi.nlm.nih.gov/pubmed/ 20613945.

15 Spinowitz B, Pecoits-Filho R, Winkelmayer WC, Pergola PE, Rochette S, ThompsonLeduc $\mathrm{P}$, et al. Economic and quality of life burden of anemia on patients with CKD on dialysis: a systematic review. J Med Econ. 2019 Jun;22(6):593-604. Available from: https:// www.ncbi.nlm.nih.gov/pubmed/30813807.

16 Sukul N, Speyer E, Tu C, Bieber BA, Li Y, Lopes AA, et al.; CKDopps and CKD-REIN investigators. Pruritus and Patient Reported Outcomes in Non-Dialysis CKD. Clin J Am Soc Nephrol. 2019 May;14(5):673-81. Available from: https://www.ncbi.nlm.nih.gov/ pubmed/30975656

17 Gregg LP, Hedayati SS. Pharmacologic and psychological interventions for depression treatment in patients with kidney disease. Curr Opin Nephrol Hypertens. 2020 Sep; 29(5):457-64. Available from: https://www. ncbi.nlm.nih.gov/pubmed/32701597.

18 Li PK, Garcia-Garcia G, Lui SF, Andreoli S, Fung WW, Hradsky A, et al.; World Kidney Day Steering Committee. Kidney health for everyone everywhere-from prevention to detection and equitable access to care. Kidney Int. $2020 \mathrm{Feb} ; 97(2)$ :226-32. Available from: https://www.ncbi.nlm.nih.gov/pubmed/ 31980067.

19 Kalantar-Zadeh K, Li PK. Strategies to prevent kidney disease and its progression. Nat Rev Nephrol. 2020 Mar;16(3):129-30. Available from: https://www.ncbi.nlm.nih.gov/ pubmed/32005966

20 Kalantar-Zadeh K, Wightman A, Liao S. Ensuring Choice for People with Kidney Failure - Dialysis, Supportive Care, and Hope. N Engl J Med. 2020 Jul;383(2):99-101. Available from: https://www.ncbi.nlm.nih.gov/pubmed/ 32640129. 